\title{
ON THE RELATIVELY PSEUDO-COMPLEMENT OPERATION IN FINITE RHAs
}

\author{
HO ANH MINH, HUYNH VAN NAM
}

\begin{abstract}
Refined hedge algebras were introduced and investigated by Ho \& Nam in [6-9]. It is known [9] that every refined hedge algebra (RHA, for short) with a chain of the primary generators is a distributive lattice. In this paper we restrict our consideration to finite version of RHAs (see $[7,9])$. It is shown that every finite RHA is a Heyting (pseudo-Boolean) algebra. Furthermore, some computing results for the relatively pseudo-complement operation in these algebras will be exhibited.
\end{abstract}

\section{INTRODUCTION}

In the research program initiated by Ho \& Wechler in [10], an algebraic approach to the natural structure of domains of linguistic variables was given. The main aim of the investigation is to find out an appropriate algebraic structure for fuzzy linguistic logic and approximate reasoning (Zadeh $[18,19])$.

On this approach, every linguistic domain can be interpreted as an algebraic structure called hedge algebra, say $X=(X, G, H, \leq)$, where $(X, \leq)$ is a poset, $G$ is a set of the primary generators and $H$ is a set of unary operations representing linguistic hedges under consideration.

It is well known that Boolean algebras, Heyting algebras, $M V$-algebras and so on, are algebraic versions of Boolean logic, Gödel logic, Lukasiewicz logic and other non-classical logic systems (see, e.g., $[15-17,3-5])$. In this direction, the idea of connecting abstract algebras with fuzzy logic becomes a natural demand and to play a useful role. This is by no means new (see, e.g., [12-14]). However, previous efforts to develop this idea have concentrated on investigating $[0,1]$-valued fuzzy logics, i.e. the algebraic versions of the unit interval $[0,1]$. Our motivation is different. We have tried to find a mathematical method for manipulating immediately linguistic terms, which were interpreted by fuzzy sets in the research on fuzzy linguistic logic and approximate reasoning started by Zadeh $[18,19]$. Therefore, our focus has been based on natural structure of linguistic domains.

This research project was initiated by Ho \& Wechler in [10] and further developed in a series of papers $[2,11,6-9]$. Supporting for this research direction has based on the fact that domains of linguistic variables can be embedded into a relatively well-known algebraic structure: distributive lattice.

Refined hedge algebras were introduced by Ho \& Nam in [6]. It is known $[8,9]$ that every RHA with a chain of the primary generators is a distributive lattice. Further, in [7,9], symmetrical RHAs were introduced and fundamental properties of these structure were examined. In this work we will restrict ourselves to finite versions of RHAs. It is shown that every finite RHA is a Heyting algebra, i.e. in these algebras we are able to define the relatively pseudo-complement operation satisfying some certain properties. Furthermore, some computing results for the relatively pseudo-complement operation in these algebras will be exhibited.

* The authors would like to thank Prof. Nguyen Cat Ho for his valuable suggestions and encourgement in their investigation.

* The research was supported in part by The National Program for Basic Research in Natural Sciences of Vietnam. 


\section{PRELIMINARIES}

Let $A$ be a lattice. For $a, b \in A$, an element $c \in A$ is said to be the pseudo-complement of a relative to $b$ (or: modulo $b$ ) if $c$ is the greatest element such that $a \cap c \leq b$, where $\cap$ stands for meet in $A$. The pseudo-complement of $a$ relative to $b$ is denoted, if it exists, by $a \Rightarrow b$. By definition, for any $x \in A, x \leq a \Rightarrow b$ if and only if $a \cap x \leq b$. If for any $a, b \in A$, there exists the element $a \Rightarrow b$ in $A$, then $A$ is called to be a Brouwerian lattice.

It is known that every Brouwerian lattice has the unit element (see, e.g., [16]). However, it does not, in general, have the zero element. A Brouwerian lattice with the zero-element is called a Heyting (pseudo-Boolean) algebra. Further, every Brouwerian lattice is distributive (see, e.g., $[1,16])$. It is also known [1] that if $A$ is a finite lattice then

$\left(^{*}\right) A$ is a Brouwerian lattice iff $A$ is a distributive lattice.

Now, let us consider RHAs. In the paper we attempt to keep our notation and conventions as in the previous papers [6-9]. For more details on RHAs we refer the reader to $[6,7,9]$.

Let $\chi=(X, G, L H, \leq)$ be an RHA constructed from $P N$-homogeneous hedge algebra $(H(G)$, $G, H, \leq$ ), where $G$ is a set of the primary generators, $H$ is a set of unary operations representing linguistic hedges under consideration, and $H(G)$ is the set of all elements generated from $G$ by means of hedge operations in $H$.

It is known that $H$ can be decomposed into two disjoint subsets $H^{+}$and $H^{-}$such that $H^{+}+I$ and $H^{-}+I$ are finite modular lattices satisfying the chain condition on their grades defined by the height function (see [6]), where $I$ is the identity, i.e. $I x=x$ for every $x$ in $X$, and is their zero-element.

As constructed in [6], $\mathrm{LH}^{+}+I$ and $\mathrm{LH}^{-}+I$ are distributive lattices generated from $\mathrm{H}^{+}+I$ and $H^{-}+I$, respectively, and $L H=L H^{+} \cup L H^{-} \cup\{I\}$. For simplicity of notation, in the sequel by "c" we mean either "+" or "-". With this notation we have

$$
L H^{c}+I=\bigcup_{i=0}^{l\left(H^{c}+I\right)} L H_{i}^{c},
$$

where $l\left(H^{c}+I\right)$ denotes the length of $H^{c}+I$.

Recall that for $i=1, \ldots, l\left(H^{c}+I\right)-1$, if $o\left(H_{i}^{c}\right)>1$ then $o\left(H_{i-1}^{c}\right)=o\left(H_{i+1}^{c}\right)=1$, where $o\left(H_{i}^{c}\right)$ denotes the number of elements of $H_{i}^{c}$. Furthermore, for any $h \in L H_{i}^{c}, k \in L H_{j}^{c}$ and $i<j$, then $h<k$. Let

$$
I^{c}=\left\{0,1, \ldots, l\left(H^{c}+I\right)\right\} \text { and } S I^{c}=\left\{i \in I^{c} \mid o\left(H_{i}^{c}\right)>1\right\} .
$$

It is known that, for any $i \in S I^{c}, L H_{i}^{c}$ is the free distributive lattice generated by incomparable elements of the grade $H_{i}^{c}$ in $H^{c}+I$, and, is also a sublattice of $L H^{c}+I$.

For any $h, k \in L H$, if $x \leq h x$ iff $x \geq k x$ for every $x$ in $X$ then $h$ and $k$ are said to be converse, or $h$ is converse to $k$ and vice-versa. If $x \leq h x$ iff $x \leq k x$ for every $x$ in $X$ then $h$ and $k$ are said to be compatible.

For the sake of convenience we repeat the relevant material from $[7,9]$ without proofs.

For any $x \in X$, let $L H[x]=\{h x \mid h \in L H\}, L H(x)=\left\{\delta x \mid \delta \in L H^{*}\right\}$, where $L H^{*}$ denotes the set of all strings of elements in $L H$.

Theorem 2.1. Let $\mathcal{X}=(X, G, L H, \leq)$ be an RHA. If $G$ is a chain then $X$ is a distributive lattice. Moreover, for any two incomparable elements $x$ and $y$ in $X$, then there exist two compatible hedge operations $h$ and $k$ in $L H$ and an element $w$ in $L H(a)$, for an element $a \in G$, such that both $h$ and $k$ together belong to $L H_{i}^{c}$ for an index $i \in S I^{c}$ and $x=\delta h w, y=\delta^{\prime} k w$, where $\delta, \delta^{\prime} \in L H^{*}$, and

$$
x \cup y= \begin{cases}\delta(h \vee k) w \cup \delta^{\prime}(h \vee k) w, & \text { if } h w>w \\ \delta(h \wedge k) w \cup \delta^{\prime}(h \wedge k) w, & \text { if } h w<w\end{cases}
$$




$$
x \cap y= \begin{cases}\delta(h \wedge k) w \cap \delta^{\prime}(h \wedge k) w, & \text { if } h w>w \\ \delta(h \vee k) w \cap \delta^{\prime}(h \vee k) w, & \text { if } h w<w\end{cases}
$$

where $\cup$ and $\cap$ stand for join and meet, respectively, in $\mathcal{X}$ while $\vee$ and $\wedge$ stand for join and meet, respectively, in $L H^{c}+I$.

Proposition 2.1. $\forall x \in X, L H[x]$ and $L H(x)$ are distributive sublattices of $\chi$.

Proposition 2.2. $\forall h, k \in L H_{i}^{c}$ for some $i \in S I^{c}, \forall x \in X$ such that $h x \neq k x$. Then $L H(h x)$ is lattice-isomorphic with $L H(k x)$.

\section{RESULTS}

Here and subsequently, $\chi$ stands for the RHA $\chi=(X, G, L H, \leq)$ considered as in the previous section, in which $G$ is a finite chain. Furthermore, the underlying set $X$ is defined as follows.

First, define $L H[G]=\bigcup_{a \in G} L H[a]$. Then, define $L H_{n}[G]$ for $n \geq 0$ by

$$
L H_{0}[G]=G, L H_{n+1}[G]=L H\left[L H_{n}[G]\right] .
$$

Notice that by convention made upon the identity $I$ (see [9]), it follows that

$$
G \subseteq L H_{1}[G] \subseteq L H_{2}[G] \subseteq \cdots \subseteq L H_{n}[G] \subseteq \cdots
$$

Let $p$ be an arbitrary but fixed positive integer. For any $x \in L H_{p}[G]$ and $x \notin L H_{p-1}[G]$, we define $h x=x$ for every $h \in L H$. Let $X=L H_{p}[G]$. Clearly, $\chi \chi$ is well-defined and, is a complete distributive lattice. Furthermore, it is known [16] that $\chi$ is a Heyting (pseudo-Boolean) algebra.

To simplify notation, we write $\varphi_{\mathcal{A}}$ instead of the relatively pseudo-complement operation defined on Browerian lattice $\mathcal{A}$. That is, for any $x, y \in \mathcal{A}, \varphi_{\mathcal{A}}(x, y)=\max \{z \in \mathcal{A} \mid x \wedge z \leq y\}$. In addition, if $A$ is a complete lattice then we denote by $1_{A}$ and $0_{A}$ the unit and zero element, respectively, in $A$. Similarly as in [1], we shall denote by $\breve{A}$ the dual of $A$ in the category of posets, and $\breve{\leq}$ the converse of the ordering relation $\leq$. Then $\widetilde{A}$ is also a lattice with the ordering relation $\leq$.

By definition, it is easily seen that the following holds.

Proposition 3.1. Let $A$ be a Brouwerian lattice. For any $x, y \in A$, we have

(i) $x \leq y$ iff $\varphi_{A}(x, y)=1_{A}$.

(ii) $\varphi_{A}(x, y) \geq y$.

(iii) If $x>y$ and $\varphi_{A}(x, y)>y$ then $x$ and $\varphi_{A}(x, y)$ are incomparable and $x \cap \varphi_{A}(x, y)=y$.

(iv) If $x$ and $y$ are incomparable then so are $x$ and $\varphi_{A}(x, y)$ and $x \cap \varphi_{A}(x, y)<y$.

We are now ready to establish some fundamental results for the relatively pseudo-complement operation in $\mathcal{X}$.

Proposition 3.2. Let $x=h_{n} \ldots h_{1} a, y=k_{m} \ldots k_{1} b$ be two canonical representations of $x$ and $y$ with respect to $a$ and $b$, respectively, in $\chi$, where $a, b \in G$ and $a \neq b$. Then

(i) If $a>b$ then $x>y$ and $\varphi_{x}(x, y)=y$.

(ii) If $a>b$ then $x<y$ and $\varphi_{x}(x, y)=1 x$.

Proof: The proof is straightforward.

The following theorem gives us a recursive formular for computing of the pseudo-complement operation in a sublattice $L H\left(x_{j}\right)$ of $\mathcal{X}$, for some $x_{j}$ of $\mathcal{X}$. Note that, according to $\left(^{*}\right)$ in Section 2 , it is legitimate to consider the operations $\varphi_{L H(x)}, \varphi_{L H_{i}^{c}}, \varphi_{L H_{i}^{c}}$ defined on $L H(x), L H_{i}^{c}, \breve{L H}_{i}^{c}$ respectively, for any $x$ of $\mathcal{X}$ and $i \in S I^{c}$. 
Theorem 3.1. Let $x=h_{n} \ldots h_{1} a, y=k_{m} \ldots k_{1} a$ be two canonical representations of $x$ and $y$ with respect to $a$, where $a \in G$, and $x \leq \mathbb{Z}$. Then, there exists an index $j \leq \min \{n, m\}+1$ such that $h_{i}=k_{i}$ for all $i<j$ and

$\varphi_{L H\left(x_{j}\right)}(x, y)= \begin{cases}y, & \text { if } \nexists i \in S I^{c} \text { such that } h_{j}, k_{j} \in L H_{i}^{c}, \\ \varphi_{L H\left(h x_{j}\right)}\left(h_{n} \ldots h_{j+1} h x_{j}, k_{m} \ldots k_{j+1} h x_{j}\right), & \text { if } \exists i \in S I^{c} \text { such that } h_{j}, k_{j} \in L H_{i}^{c} \\ & \text { and } h_{j} x_{j}>x_{j}, \\ \varphi_{L H\left(\breve{h} x_{j}\right)}\left(h_{n} \ldots h_{j+1} \breve{h} x_{j}, k_{m} \ldots k_{j+1} \breve{h} x_{j}\right), & \text { if } \exists i \in S I^{c} \text { such that } h_{j}, k_{j} \in L H_{i}^{c} \\ & \text { and } h_{j} x_{j}<x_{j},\end{cases}$

where $x_{j}=h_{j-1} \ldots h_{1} a$, and

$$
h= \begin{cases}\varphi_{L H_{i}^{+}}\left(h_{j}, k_{j}\right), & \text { if } i \in S I^{+} \\ \varphi_{\breve{L H}_{i}^{-}}\left(h_{j}, k_{j}\right), & \text { if } i \in S I^{-}\end{cases}
$$

and

$$
\breve{h}= \begin{cases}\varphi_{L H_{i}^{+}}\left(h_{j}, k_{j}\right), & \text { if } i \in S I^{+} \\ \varphi_{L H_{i}^{-}}\left(h_{j}, k_{j}\right), & \text { if } i \in S I^{-}\end{cases}
$$

Notice that $L H_{i}^{c}$ is a sublattice of $L H^{c}+I$, while $\breve{L H_{i}^{c}}$ is a sublattice of $L H^{c}+I$.

Proof: Let $j$ be the least index such that $h_{j} \neq k_{j}$. If $\nexists i \in S I^{c}$ such that $h_{j}, k_{j} \in L H_{i}^{c}$ then it implies that $x$ and $y$ are comparable, and hence $x>y$ and $h_{j} x_{j}>k_{j} x_{j}$, since $x \not \leq y$. Assume that $h_{j} \in L H_{i_{0}}^{c}$ for some $i_{0} \in I^{c}$. If $\varphi_{L H\left(x_{j}\right)}(x, y)>y$ then it follows from (iii) of Proposition 3.1 that $x$ and $\varphi_{L H\left(x_{j}\right)}(x, y)$ are incomparable. Thus, there exists $h^{\prime} \in L H_{i_{0}}^{c}$ such that $\varphi_{L H\left(x_{j}\right)}(x, y) \in L H\left(h^{\prime} x_{j}\right)$. Hence, there exists $h^{\prime \prime} \in L H_{i_{0}}^{c}$ such that $\left(x \cap \varphi_{L H\left(x_{j}\right)}(x, y)\right) \in L H\left(h^{\prime \prime} x_{j}\right)$. Since $k_{j} \notin L H_{i_{0}}^{c}$ and $h_{j} x_{j}>k_{j} x_{j}$, it follows that $x \cap \varphi_{L H\left(x_{j}\right)}(x, y)>y$, which is impossible. So we infer $\varphi_{L H\left(x_{j}\right)}(x, y)=y$.

Now suppose that there exists $i \in S I^{c}$ such that $h_{j}, k_{j} \in L H_{i}^{c}$ and $h_{j} x_{j}>x_{j}$. By the properties of $\mathcal{X}$, it is easily seen that $\varphi_{L H\left(x_{j}\right)}(x, y) \in L H\left(h^{\prime} x_{j}\right)$ for some $h^{\prime} \in L H_{i}^{c}$. Assume that $\varphi_{L H\left(x_{j}\right)}(x, y)=\delta h^{\prime} x_{j}$ for some $\delta \in L H^{*}$. Then we have $\delta h^{\prime} x_{j} \cap x \leq y$, i.e. $\delta h^{\prime} x_{j} \cap h_{n} \ldots h_{j} x_{j} \leq$ $k_{m} \ldots k_{j} x_{j}$. It follows from Theorem 2.1. that

$$
\delta\left(h^{\prime} \wedge h_{j}\right) x_{j} \cap h_{n} \ldots h_{j+1}\left(h^{\prime} \wedge h_{j}\right) x_{j} \leq k_{m} \ldots k_{j} x_{j} .
$$

Hence, it implies that $\left(h^{\prime} \wedge h_{j}\right) x_{j} \leq k_{j} x_{j}$. Again by Theorem 2.1 we obtain $h^{\prime} x_{j} \cap h_{j} x_{j} \leq k_{j} x_{j}$. Let

$$
h= \begin{cases}\varphi_{L H_{i}^{+}}\left(h_{j}, k_{j}\right), & \text { if } i \in S I^{+} \\ \varphi_{L H_{i}^{-}}\left(h_{j}, k_{j}\right), & \text { if } i \in S I^{-}\end{cases}
$$

then by the definition of $h$ and the last inequatily we infer

$$
h^{\prime} x_{j} \leq h x_{j} .
$$

On the other hand, we have

$$
\delta h x_{j} \cap x=\delta\left(h \wedge h_{j}\right) x_{j} \cap h_{n} \ldots h_{j+1}\left(h \wedge h_{j}\right) x_{j} .
$$

From Proposition 2.2 and (1) it is easy to check that

$$
\delta k_{j} x_{j} \cap h_{n} \ldots h_{j+1} k_{j} x_{j} \leq k_{m} \ldots k_{j} x_{j} .
$$


Once again, since $\left(h \wedge h_{j}\right) x_{j} \leq k_{j} x_{j}$, it follows from Proposition 2.2 and (4) that

$$
\delta\left(h \wedge h_{j}\right) x_{j} \cap h_{n} \ldots h_{j+1}\left(h \wedge h_{j}\right) x_{j} \leq k_{m} \ldots k_{j+1}\left(h \wedge h_{j}\right) x_{j} \leq k_{m} \ldots k_{j} x_{j} .
$$

From the last inequalities and (3), we obtain $\delta h x_{j} \cap x \leq y$, and hence $\delta h x_{j} \leq \delta h^{\prime} x_{j}$. Thus, we have $h x_{j} \leq h^{\prime} x_{j}$. Since $x_{j}$ does not be a fixed point and by (2) it implies $h^{\prime}=h$. Further, it follows again by Proposition 2.2 and (4) that

$$
\delta h x_{j} \cap h_{n} \ldots h_{j+1} h x_{j} \leq k_{m} \ldots k_{j+1} h x_{j} .
$$

Therefore, we have

$$
\varphi_{L H\left(x_{j}\right)}(x, y)=\delta h x_{j} \leq \varphi_{L H\left(h x_{j}\right)}\left(h_{n} \ldots h_{j+1} h x_{j}, k_{m} \ldots k_{j+1} h x_{j}\right) .
$$

Now assume that $\varphi_{L H\left(h x_{j}\right)}\left(h_{n} \ldots h_{j+1} h x_{j}, k_{m} \ldots k_{j+1} h x_{j}\right)=\delta^{\prime} h x_{j}$, for some $\delta^{\prime} \in L H^{*}$. Since $\delta^{\prime} h x_{j} \cap h_{n} \ldots h_{j+1} h x_{j} \leq k_{m} \ldots k_{j+1} h x_{j}$, we have

$$
\delta^{\prime}\left(h \wedge h_{j}\right) x_{j} \cap h_{n} \ldots h_{j+1}\left(h \wedge h_{j}\right) x_{j} \leq k_{m} \ldots k_{j+1}\left(h \wedge h_{j}\right) x_{j}
$$

or

$$
\delta^{\prime} h x_{j} \cap h_{n} \ldots h_{j} x_{j} \leq k_{m} \ldots k_{j+1}\left(h \wedge h_{j}\right) x_{j} \leq k_{m} \ldots k_{j} x_{j},
$$

and hence $\delta^{\prime} h x_{j} \cap x \leq y$. We thus get

$$
\delta^{\prime} h x_{j} \leq \delta h x_{j}=\varphi_{L H\left(x_{j}\right)}(x, y) .
$$

From (5) and (6), we obtain the desired equality

$$
\varphi_{L H\left(x_{j}\right)}(x, y)=\varphi_{L H\left(h x_{j}\right)}\left(h_{n} \ldots h_{j+1} h x_{j}, k_{m} \ldots k_{j+1} h x_{j}\right) .
$$

The proof for the remain case can be obtained by a similar argument. Consequently, the proof is complete.

Now the main result is this. The following theorem establishes a recursive formula for computing the pseudo-complement operation in $\chi$.

Theorem 3.2. Under the same hypothesises and notation as in Theorem 9.1, we have

$$
\varphi_{x}(x, y)=\left\{\begin{array}{c}
\varphi_{L H\left(x_{j}\right)}(x, y), \text { if } h_{i} \in \underset{s \in\left(I^{c} \backslash S I^{c}\right), c \in\{+,-\}}{U} L H_{s}^{c} \text { for any } i \leq j-1, \\
\varphi_{L H\left(h x_{t}\right)}\left(h_{n} \ldots h_{j} h_{j-1} \ldots h_{t+1} h x_{t}, k_{m} \ldots k_{j} h_{j-1} \ldots h_{t+1} h x_{t}\right), \text { if there exists the } \\
\text { least index } t \text { such that } h_{t} \in L H_{i_{t}}^{c} \text { for some } i_{t} \in S I^{c} \text { and } h_{t} x_{t}>x_{t}, \\
\varphi_{L H\left(\breve{h} x_{t}\right)}\left(h_{n} \ldots h_{j} h_{j-1} \ldots h_{t+1} \breve{h} x_{t}, k_{m} \ldots k_{j} h_{j-1} \ldots h_{t+1} h x_{t}\right), \text { if there exists the } \\
\text { least index } t \text { such that } h_{t} \in L H_{i_{t}}^{c} \text { for some } i_{t} \in S I^{c} \text { and } h_{t} x_{t}<x_{t},
\end{array}\right.
$$

where $x_{t}=h_{t-1} \ldots h_{1} a$, and

$$
h= \begin{cases}1_{L H_{i_{t}}^{+}}, & \text {if } i_{t} \in S H^{+} \\ 0_{L H_{i_{t}}^{-}}, & \text {if } i_{t} \in S I^{-}\end{cases}
$$

and

$$
\breve{h}= \begin{cases}0_{L H_{i_{t}}^{+}}, & \text {if } i_{t} \in S H^{+} \\ 1_{L H_{i_{t}}^{-}}, & \text {if } i_{t} \in S I^{-}\end{cases}
$$


Proof: For the case where $h_{i} \in \bigcup_{s \in\left(I^{c} \backslash S I^{\circ}\right), c \in\{+,-\}} L H_{s}^{c}$ for any $i \leq j-1$, it is easy to check that $\varphi_{X}(x, y)=\varphi_{L H\left(x_{j}\right)}(x, y)$, by the assumptions made upon elements $x$ and $y$ and the definition of RHA.

In the opposite case, let $t$ be the least index satisfying $t \leq j-1$ such that $h_{t} \in L H_{i_{t}}^{c}$, for some $i_{t} \in S I^{c}$. Assume first that $h_{t} x_{t}>x_{t}$, then we have $h x_{t} \geq h_{t} x_{t}$. By the assumption that $x \not \leq y$ and Proposition 2.2 we get

$$
h_{n} \ldots h_{t+1} h x_{t} \not k_{m} \ldots k_{t+1} h x_{t},
$$

with a notice that $h_{i}=k_{i}$ for any $i$ such that $t+1 \leq i \leq j-1$. that

Let $\varphi_{L H\left(h x_{t}\right)}\left(h_{n} \ldots h_{t+1} h x_{t}, k_{m} \ldots k_{t+1} h x_{t}\right)=\delta h x_{t}$. It follows immediately from Theorem 2.1

$$
x \cap \delta h x_{t}=x \cap \delta h_{t} x_{t},
$$

since $h_{t} x_{t}>x_{t}$. In addition, since

$$
h_{n} \ldots h_{t+1} h x_{t} \cap \delta h x_{t} \leq k_{m} \ldots k_{t+1} h x_{t}
$$

it implies by Proposition 2.2 that

$$
x \cap \delta h_{t} x_{t} \leq y .
$$

We infer from (7) and (8) that $x \cap \delta h x_{t} \leq y$. This shows that

$$
\varphi x(x, y) \geq \delta h x_{t} .
$$

On the other hand, by the definition of RHA and Proposition 3.1, it can easily be seen that $\varphi_{x}(x, y) \in L H\left(k x_{t}\right)$ for some $k \in L H_{i_{t}}^{c}$. Let $\varphi_{x}(x, y)=\delta^{\prime} k x_{t}$. By (9) and the definition of $h$, it is easy to check that $h=k$. Further, by definition we have $x \cap \delta^{\prime} h x_{t} \leq y$. This shows that

$$
x \cap \delta^{\prime} h_{t} x_{t} \leq y,
$$

by Theorem 2.1. From (10) and Proposition 2.2 we infer

$$
h_{n} \ldots h_{t+1} h x_{t} \cap \delta^{\prime} h x_{t} \leq k_{m} \ldots k_{t+1} h x_{t}
$$

or

$$
\delta^{\prime} h x_{t} \leq \delta h x_{t}=\varphi_{L H\left(h x_{t}\right)}\left(h_{n} \ldots h_{t+1} h x_{t}, k_{m} \ldots k_{t+1} h x_{t}\right) .
$$

Thus $\varphi_{x}(x, y)=\varphi_{L H\left(h x_{t}\right)}\left(h_{n} \ldots h_{t+1} h x_{t}, k_{m} \ldots k_{t+1} h x_{t}\right)$, by (9) and (11). For the case where $h_{t} x_{t}<x_{t}$, the proof is similar. Consequently, the theorem is completely proved.

\section{REFERENCES}

[1] G. Birkhoff, Lattice Theory, Providence, Rhode Island, 1973.

[2] N. Cat Ho, A method in linguistic reasoning on a knowledge base representing by sentences with linguistic belief degree, Fundamenta Informaticae $28(3,4)(1996)$ 247-259.

[3] N. Cat Ho, H. Rasiowa, semi-Post algebras and their representability, Studia Logica 48 (4) (1989) 509-530.

[4] N. Cat Ho, H. Rasiowa, LT-Fuzzy sets, Fuzzy Sets and Systems 47 (1992) 323-339.

[5] N. Cat Ho, H. Rasiowa, LT-Fuzzy Logics, in L. A. Zadeh and J. Kacprzyk, Eds., Fuzzy Logic for Management of Uncertainty, Wiley, New York, 1992.

[6] N. Cat Ho, H. Van Nam, A refinement structure of hedge algebras, Proc. of the NCST of Vietnam 9 (1) (1997) 15-28. 
[7] N. Cat Ho, H. Van Nam, Symmetrical RHA and its application to fuzzy logic, to appear in Proc. of the NCST of Vietnam.

[8] N. Cat Ho, H. Van Nam, Refinement structure of hedge algebras: An algebraic basis for a linguistic-valued fuzzy logic, Presented at Inter. Conf. on Discrete Mathematics and Allied Topics, 10-13 Nov. 1997, India.

[9] N. Cat Ho, H. Van Nam, A theory of refinement structure of hedge algebras and its application to linguistic-valued fuzzy logic, In D. Niwinski \& M. Zawadowski, Eds., "Logic, Algebra and Computer Science" (Banach Center Publications, PWN - Polish Scientific Publishers, Warszawa, 1998).

[10] N. Cat Ho, W. Wechler, Hedge algebras: An algebraic approach to structure of sets of linguistic truth values, Fuzzy Sets and Systems $\mathbf{3 5}$ (1990) 281-293.

[11] N. Cat Ho, W. Wechler, Extended hedge algebras and their application to fuzzy logic, Fuzzy Sets and Systems 52 (1992) 259-281.

[12] D. Dumitrescu, Fuzzy conditional logic, Fuzzy Sets and Systems 68 (1994) 171-179.

[13] E. P. Klement, Some mathematical aspects of fuzzy sets: Triangular norms, fuzzy logics, and generalized measures, Fuzzy Sets and Systems 90 (1997) 133-140.

[14] H. T. Nguyen, V. Kreinovich, Fuzzy logic as applied linear logic, BUSEFAL (1996) (IRIT, Uni. P. Sabatier, Toulouse, France).

[15] H. Rasiowa, An Algebraic Approach to Non-classical Logic, North-Holland, Amsterdam - New York, 1974.

[16] H. Rasiowa, R. Sikorski, The Mathematics of Metamathematics, second edition, Polish Scientific Publ., Warszawa, 1968.

[17] R. Sikorski, Boolean Algebras, third edition, Springer-Verlag, Berlin-Heidelberg-New York, 1969.

[18] L. A. Zadeh, A theory of approximate reasoning, in: J. E. Hayes, D. Mitchie, and L. I. Mikulich, Eds., Machine Intelligence, Vol.9, Wiley, New York, 1979, 149-194.

[19] L. A. Zadeh, The concept of linguistic variable and its application to approximate reasoning (I) \& (II), Inform. Sci. 8 (1975) 199-249 \& 8 (1975) 310-357.

Tóm tắt. Để xây dựng một cơ sở đại số cho logic mờ theo nghĩa của Zadeh, đại số gia tử mịn hóa đã được giói thiệu và nghiên cứu trong [6-9]. Trong [9], các tác giả đã chứng minh rằng đại số gia tử mịn hóa với dây chuyền các phần tử sinh nguyên thưy là một dàn phân phối. Trong bài báo này chúng tôi hạn chế xem xét trên các đại số gia tứ mịn hóa hữu hạn. Khi đó đại số gia tử mịn hóa trở thành một đại số Heyting, tức là trong các đại số gia tứ mịn hóa hữu hạn chúng ta có thể định nghĩa toán tử tựa phần bù tương đối thỏa mãn một số tính chất xác định (xem $[1,16])$. Chúng tôi cũng đưa ra các kết quả tính toán cho toán tử đó trong các đại số gia tử mịn hóa hữu hạn.

Department of Mathematics

Received: January 20, 1998

Qui Nhon University of Pedagogy 